\title{
Fuzzy probability distribution with VaR constraint for portfolio selection
}

\author{
M. Rocha ${ }^{1}$ L. Lima ${ }^{2}$ H. Santos ${ }^{3}$ B. Bedregal ${ }^{4}$ \\ ${ }^{1}$ Graduate program in Mathematics and statistics, UFPA, Belem, Brasil \\ ${ }^{2}$ Graduate program in Electrical Engineering and Computing,UFRN, Natal, Br \\ ${ }^{3}$ Department of Informatics and Applied Mathematics, UFRN, Natal, Br \\ ${ }^{4}$ Department of Informatics and Applied Mathematics, UFRN, Natal, Br
}

\begin{abstract}
This work aims at comparing two models of fuzzy distribution: Normal and Laplace, whenever they are inside the context of possibilistic mean-variance model described by Li et al. in [6], where fuzzy Normal distribution is used. We propose to make a comparison using their model, but instead we apply fuzzy Laplace distribution. We also demonstrate the theorems which are necessary for the inclusion of these distributions to the model proposed by $\mathrm{Li}$ et al. So, we evaluate the behavior of this model when these distribution functions are changed and we also vary the VaR (Value at Risk). For financial analysts it is very important having other distributions as parameters, regarding the volatility of the stock market due to the behavior of financial market.
\end{abstract}

Keywords: Number Fuzzy, VaR, Portfolio selection, Risk

\section{Introduction}

The least complex and most natural way to represent the problem of optimal portfolio selection is a constrained optimization problem. The aim is to maximize or minimize an objective function (usually maximize returns or risk minimization) subject to constraints. However, the objective function and constraints are usually not simple functions. They often rely on more than one characteristic of each asset, and these characteristics are usually combinations of functions that are much more complex than a linear or quadratic function. So, finding a solution to this optimization problem requires more complex techniques.

For these and other reasons, many researchers seek models that can measure all of these variables. In this sense, Markowitz [7],[8] proposed a model for the mean-variance portfolio selection and probability theory associated to optimization techniques to model the performance of investment under uncertainty.

However, due to the complexity of financial systems, there are several situations where the input data are not precise but only fuzzy. Therefore, the decision makers should not consider parameters (goals and constraints) using numbers or unique distribuition functions, but instead they should use fuzzy numbers or fuzzy probability distribution functions (see, for example, Zdenek Zmeskal [16]). Recently, researchers investigated many fuzzy portfolio selection problems (see, e.g., Watada [13], Ramaswamy [9], Leon et al. [5], Wang and Zhu [12], Tanaka et al. [11] and Zhang et al. [17]). Carlsson and Fuller [2] introduced the notations ââof upper and lower possibilistic mean values, and introduced the notation of crisp possibilistic mean values and crisp possibilistic variance of continuous distributions. Zhang and Nie [18] extended the concepts of possibilistic mean and possibilistic variance proposed by Carlsson and Fuller [2], and introduced the concepts of upper and lower possibilistic variances and covariances of fuzzy numbers.

We propose to make a comparison using their model, but instead we apply fuzzy Laplace. We also demonstrate the theorems which are necessary for the inclusion of these distributions to the model proposed by Li et al. So, we evaluate the behavior of this model when these distributions functions are changed and we also vary the VaR (Value at Risk).

This paper is organized as follows. In section 2, we introduce the basic concepts of the possibilistic mean and variance of a fuzzy number. In section 3 , we proposed a possibilistic portfolio model under constraints of VaR and risk-free investment. In section 4 , we present a fuzzy normal distribution demonstrated in $\mathrm{Li}[6]$. In section 5, we present fuzzy Laplce distribution. In Section 6, numerical examples are given to illustrate our effective proposed approaches. And finally, Section 7 presents our conclusions.

\section{Possibilistic variance and mean value}

In this section, we introduce some concepts that will be used in the next sections. A fuzzy number $\tilde{A}$ is a fuzzy set of the real line $\mathbb{R}$ with a normal, convex and continuous membership function of bounded support. The family of fuzzy number will be denoted by $F$. A $\gamma$-level set of fuzzy $\tilde{A}$ is defined by $[\tilde{A}]^{\gamma}=\{t \in \mathbb{R} \mid \tilde{A}(t) \geq \gamma\}$ if $\gamma>0$ and $[\tilde{A}]^{\gamma}=\operatorname{cl}\{t \in \mathbb{R} \mid \tilde{A}(t)>0\}$ (the closure of the sup- 
port of $\tilde{A}$ ) if $\gamma=0$. It is well known that if $\tilde{A}$ is a fuzzy number then $[\tilde{A}]^{\gamma}$ is a compact subset of $\mathbb{R}$ for all $\gamma \in[0,1]$ and $[\tilde{A}]^{\gamma}=\left[a_{1}(\gamma), a_{2}(\gamma)\right]$.

Carlsson and Fuller [2] defined the upper and lower possibilistic means of a fuzzy number $\tilde{A}$. First, we can define $\bar{M}(\tilde{A})$ as

$$
\bar{M}(\tilde{A})=\int_{0}^{1} \gamma\left(a_{1}(\gamma)+a_{2}(\gamma)\right) d \gamma .
$$

We can rewrite $\bar{M}(\tilde{A})$ as

$$
\bar{M}(\tilde{A})=\frac{1}{2}\left(\frac{\int_{0}^{1} \gamma\left(a_{1}(\gamma)\right) d \gamma}{\frac{1}{2}}+\frac{\int_{0}^{1} \gamma\left(a_{2}(\gamma)\right) d \gamma}{\frac{1}{2}}\right),
$$

or

$$
\bar{M}(\tilde{A})=\frac{1}{2}\left(\frac{\int_{0}^{1} \gamma\left(a_{1}(\gamma)\right) d \gamma}{\int_{0}^{1} \gamma d \gamma}+\frac{\int_{0}^{1} \gamma\left(a_{2}(\gamma)\right) d \gamma}{\int_{0}^{1} \gamma d \gamma}\right) .
$$

Therefore,

$$
\bar{M}(\tilde{A})=\frac{\int_{0}^{1} \gamma\left(a_{1}(\gamma)+a_{2}(\gamma)\right) / 2 d \gamma}{\int_{0}^{1} \gamma d \gamma} .
$$

It follows that $\bar{M}(\tilde{A})$ is nothing but the levelweighted average of the arithmetic means of all $\gamma$ level sets, that is, the weight of the arithmetic mean of $a_{1}(\gamma)$ and $a_{2}(\gamma)$ is just $\gamma$.

Lets look at the right-hand side of equation (4). The first quantity, denoted by $M_{U}(\tilde{A})$ can be reformulated as

$$
M_{U}(\tilde{A})=\frac{\int_{0}^{1} \operatorname{Pos}\left[\tilde{A} \leq a_{1}(\gamma)\right]\left(a_{1}(\gamma)\right) d \gamma}{\int_{0}^{1} \operatorname{Pos}\left[\tilde{A} \leq a_{1}(\gamma)\right] d \gamma},
$$

where Pos denotes possibility, i.e.

$$
\operatorname{Pos}\left[\tilde{A} \leq a_{1}(\gamma)\right]=\underbrace{\sup }_{u \leq a_{1}(\gamma)} A(u)=\gamma .
$$

Similarly, we can denote $M_{L}(\tilde{A})$ as

$$
M_{L}(\tilde{A})=\frac{\int_{0}^{1} \operatorname{Pos}\left[\tilde{A} \geq a_{2}(\gamma)\right]\left(a_{2}(\gamma)\right) d \gamma}{\int_{0}^{1} \operatorname{Pos}\left[\tilde{A} \geq a_{2}(\gamma)\right] d \gamma}
$$

where Pos denotes possibility, i.e.

$$
\operatorname{Pos}\left[\tilde{A} \geq a_{2}(\gamma)\right]=\underbrace{\text { sup }}_{u \geq a_{2}(\gamma)} A(u)=\gamma
$$

The possibilistic mean value of $\tilde{A}$ is the arithmetic mean of its lower and upper possibilistic mean values as follows:

$$
\bar{M}(\tilde{A})=\frac{M_{U}(\tilde{A})+M_{L}(\tilde{A})}{2}
$$

Similarly as shown for the upper and lower possibilistic means, Zhang and Nie [17] introduced the upper and lower possibilistc variances and covariances fuzzy numbers. The upper and lower possibilistic variances of a fuzzy set $\tilde{A}$ is defined as:

$$
\sigma_{U}^{2}(\tilde{A})=2 \int_{0}^{1} \gamma\left(M_{U}(\tilde{A})-a_{2}(\gamma)\right)^{2} d \gamma,
$$

and

$$
\sigma_{L}^{2}(\tilde{A})=2 \int_{0}^{1} \gamma\left(M_{L}(\tilde{A})-a_{1}(\gamma)\right)^{2} d \gamma
$$

respectively.

The upper and lower possibilistic covariances between fuzzy numbers, are defined, respectively, as:

$\operatorname{Cov}_{U}(\tilde{A}, \widetilde{B})=2 \int_{0}^{1} \gamma\left(M_{U}(\tilde{A})-a_{2}(\gamma)\right)\left(M_{U}(\widetilde{B})-b_{2}(\gamma)\right) d \gamma$

and

$\operatorname{Cov}_{L}(\tilde{A}, \widetilde{B})=2 \int_{0}^{1} \gamma\left(M_{L}(\tilde{A})-a_{1}(\gamma)\right)\left(M_{L}(\widetilde{B})-b_{1}(\gamma)\right) d \gamma$

The possibilistic variance of fuzzy number $\tilde{A}$ is defined as:

$$
\overline{\sigma^{2}}(\tilde{A})=\frac{\sigma_{U}^{2}(\tilde{A})+\sigma_{L}^{2}(\tilde{A})}{2} .
$$

The possibilistic covariance between the fuzzy numbers $\widetilde{A}$ and $\widetilde{B}$ is defined as:

$$
\overline{\operatorname{Cov}}(\widetilde{A}, \widetilde{B})=\frac{\operatorname{Cov}_{U}(\widetilde{A}, \widetilde{B})+\operatorname{Cov}_{L}(\widetilde{A}, \widetilde{B})}{2}
$$

Lemma 2.1 [2] Let $\widetilde{A}$ and $\widetilde{B}$ be fuzzy numbers. Then for each $\lambda_{1}, \lambda_{2} \in \mathbb{R}$,

$$
\bar{M}\left(\lambda_{1} \widetilde{A}+\lambda_{2} \widetilde{B}\right)=\lambda_{1} \bar{M}(\widetilde{A})+\lambda_{2} \bar{M}(\widetilde{B}) .
$$

Lemma 2.2 [2] Let $\widetilde{A}$ and $\widetilde{B}$ be fuzzy numbers. Then for each $\lambda_{1}, \lambda_{2} \in \mathbb{R}$,

$$
\begin{array}{r}
\overline{\sigma^{2}}\left(\lambda_{1} \widetilde{A}+\lambda_{2} \widetilde{B}\right)=\lambda_{1}^{2} \overline{\sigma^{2}}(\widetilde{A})+\lambda_{2}^{2} \overline{\sigma^{2}}(\widetilde{B}) \\
+2\left|\lambda_{1} \lambda_{2}\right| \overline{\operatorname{Cov}}\left(\phi\left(\lambda_{1}\right) \widetilde{A}, \phi\left(\lambda_{2}\right) \widetilde{B}\right),
\end{array}
$$

where $\phi(x)$ is a sign function of $x \in \mathbb{R}$.

\section{Portfolio Model under constraints of VaR and risk free investment}

In order to define the model, it is necessary to make the following considerations. First, there are $n$ risk assets and one risk-free asset for investment and the asset return rate $\widetilde{\varphi}_{i}$ is a fuzzy number, $i=1,2, \ldots, n . \quad x_{i}$ represents the proportion invested in assets $i$, and $r_{f}$ is the risk-free asset return. From this, we can then define the return $\widetilde{r_{p}}$ as 


$$
\widetilde{r_{p}}=\sum_{i=1}^{n} x_{i} \widetilde{\varphi}_{i}+r_{f}\left(1-\sum_{i=1}^{n} x_{i}\right)
$$

since $\widetilde{\varphi}_{i}$ is a fuzzy membership $\widetilde{r_{p}}$ is also a fuzzy number.

Consequently, the possibilistic mean of the portfolio return $\widetilde{r_{p}}$ is

$$
\bar{M}\left(\widetilde{r_{p}}\right)=\sum_{i=1}^{n} x_{i} \frac{M_{U}\left(\widetilde{\varphi_{i}}\right)+M_{L}\left(\widetilde{\varphi_{i}}\right)}{2}+r_{f}\left(1-\sum_{i=1}^{n} x_{i}\right) .
$$

According to Lemma 2.2, it is known that the possibilistic variance of $\widetilde{r_{p}}$ is given by

$$
\overline{\sigma^{2}}\left(\sum_{i=1}^{n} x_{i} \widetilde{\varphi_{i}}\right)=\sum_{i=1}^{n} x_{i}^{2} \overline{\sigma_{\varphi_{i}}^{2}}+2 \sum_{i>j=1}^{n} x_{i} x_{j} \overline{\operatorname{Cov}}\left(\widetilde{\varphi_{i}}, \widetilde{\varphi_{j}}\right)
$$

A value at risk VaR constraint is imposed on our portfolio model, as follows. Replacing equation (6) and considering $a_{1}(\gamma)=\operatorname{VaR} \leq 1-\beta$ and $\beta$ as confidence level, we obtain

$$
\operatorname{Pos}[\tilde{A} \leq \operatorname{VaR})]=\underbrace{\text { sup }}_{t \geq V a R} A(t) \leq 1-\beta .
$$

Finally, we can define the possibilistic meanvariance model regarding the possibilistic mean as the portfolio return and the possibilistic variance as the portfolio risk. Under this structure, the possibilistic portfolio model under constraint of VaR and risk-free investment can be formulated as:

$$
\left\{\begin{array}{cc}
\min & \overline{\sigma^{2}}=\sum_{i=1}^{n} x_{i}^{2} \overline{\sigma_{\widetilde{\varphi}}^{2}}+2 \sum_{i>j=1}^{n} x_{i} x_{j} \overline{\operatorname{Cov}}\left(\widetilde{\varphi_{i}}, \widetilde{\varphi_{j}}\right) \\
\text { s.t. } & \sum_{i=1}^{n} x_{i} \frac{M_{U}\left(\widetilde{\varphi_{i}}\right)+M_{L}\left(\widetilde{\varphi_{i}}\right)}{2}+r_{f}\left(1-\sum_{i=1}^{n} x_{i}\right) \geq \widetilde{r} \\
& \operatorname{pos}\left(\widetilde{\left.\varphi_{i} x_{i} \leq V a R\right) \leq 1-\beta,}\right. \\
& \sum_{i=1}^{n} x_{i} \leq 1, \\
0 \leq l_{i} \leq x_{i} \leq u_{i}, i=1,2, \ldots, n,
\end{array}\right.
$$

where pos denotes the measure of possibilistic, $\widetilde{r}$ is the underestimated expected rate of return, $l_{i}$ and $u_{i}$ represent the lower bound and upper bound on investment in asset $i$, respectively, and $\mathrm{VaR}$ is defined as the value at risk by the $\beta$-confidence level.

The model shows that risk-averse investors wish not only to reach the expected rate of returns in their actual investment, but also to ensure that the maximum of their possible risk is lower than an expected loss.

\section{Fuzzy Normal Distribution}

According to $\mathrm{Li}[6]$ the rate of return on asset $i$ is a fuzzy variable with fuzzy normal distribution expressed by $\widetilde{\xi}_{i} \sim F N\left(\mu_{i}, \sigma_{i}\right)$, and its membership function is

$$
A_{\widetilde{\xi}_{i}}(t)=\exp \left\{-\left[\left(t-\mu_{i}\right) / \sigma_{i}\right]^{2}\right\} .
$$

The $\gamma$-level set of $\widetilde{\xi}$ is

$$
\begin{array}{r}
{\left[\widetilde{\xi}_{i}\right]^{\gamma}=\left[\mu_{i}-\sigma_{i} \sqrt{\ln \gamma^{-1}}, \mu_{i}+\sigma_{i} \sqrt{\ln \gamma^{-1}}\right],} \\
\gamma \in(0,1), i=1,2, . ., n .
\end{array}
$$

Theorem 4.1 Assume that the return rates of assets are fuzzy variables with fuzzy normal distribution expressed as $\widetilde{\varphi}_{i} \sim F N\left(\mu_{i}, \sigma_{i}\right), i=1,2, \ldots, n$, then

$\sum_{i=1}^{n} x_{i} \widetilde{\varphi_{i}} \sim F N\left[\sum_{i=1}^{n} x_{i} \mu_{i},\left(\frac{1}{2}-\frac{\pi}{8}\right)\left(\sum_{i=1}^{n} x_{i} \sigma_{i}\right)^{2}\right]$,

where $x_{i} \geq 0, i=1,2, \ldots, n$.

\section{Proof}

Refer to [6].

Moreover, Li [6] defines the possibilistic portfolio model under constraints of VaR and risk-free investments, whereas the variables are fuzzy with fuzzy Normal distribution. Thus:

$$
\left\{\begin{array}{c}
\min \overline{\sigma^{2}}=\left(\frac{1}{2}-\frac{\pi}{8}\right)\left(\sum_{i=1}^{n} x_{i}^{2} \sigma_{2}^{2}+2 \sum_{1>j=1}^{n} x_{i} x_{j} \sigma_{i} \sigma_{j}\right) \\
\text { s.t. } \quad \sum_{i=1}^{n} x_{i}\left(\mu_{i}-r_{f}\right)+r_{f} \geq \widetilde{r} \\
-\left(V a R-\sum_{i=1}^{n} x_{i} \mu_{i}\right)^{2} \leq \ln (1-\beta)\left(\frac{1}{2}-\frac{\pi}{8}\right)\left(\sum_{i=1}^{n} x_{i} \sigma_{i}\right)^{2} \\
\quad \sum_{i=1}^{n} x_{i} \leq 1 \\
0 \leq l_{i} \leq x_{i} \leq u_{i}, i=1,2, \ldots, n .
\end{array}\right.
$$

\section{Fuzzy Laplace Distribution}

Suppose that the return rate of asset $i$ is a Laplace distribution fuzzy variable expressed as $\widetilde{\varphi}_{i} \sim$ $F L\left(\mu_{i}, \sigma_{i}\right)$, and its membership function is

$$
\begin{aligned}
A_{\tilde{\varphi}_{i}}\left(t / \mu_{i}, b\right) & =\frac{1}{2 b} \exp \left(-\frac{\left|t-\mu_{i}\right|}{b}\right) \\
& =\frac{1}{2 b}\left\{\begin{array}{l}
\exp \left(\frac{-\mu_{i}+t}{b}\right) \text { if } t<\mu_{i} \\
\exp \left(\frac{-t+\mu_{i}}{b}\right) \text { if } t \geq \mu_{i},
\end{array}\right.
\end{aligned}
$$

where $\sigma_{i}^{2}=2 b^{2} \Rightarrow b=\frac{\sqrt{2}}{2} \sigma_{i}$.

The $\gamma$-level set of $\widetilde{\varphi}_{i}$ is defined as

$$
\left[\widetilde{\varphi}_{i}\right]^{\gamma}=\left[\mu_{i}-\frac{\sqrt{2}}{2} \sigma_{i} \ln \sqrt{2} \sigma_{i} \gamma, \mu_{i}+\frac{\sqrt{2}}{2} \sigma_{i} \ln \sqrt{2} \sigma_{i} \gamma\right] .
$$

Theorem 5.1 We assume that the rates of return of the assets are Laplace fuzzy distributions variables expressed as $\widetilde{\varphi}_{i} \sim F L\left(\mu_{i}, \sigma_{i}\right), i=1,2, \ldots, n$. Then

$$
\sum_{i=1}^{n} x_{i} \widetilde{\varphi}_{i} \sim F L\left(\sum_{i=1}^{n} x_{i} \mu_{i}, \frac{1}{8}\left(\sum_{i=1}^{n} x_{i} \sigma_{i}\right)^{2}\right)
$$

where $x_{i} \geq 0, i=1,2, \ldots, n$.

\section{Proof}

According to Lemma 2.1, the possibilistic mean value of $\sum_{i=1}^{n} x_{i} \widetilde{\varphi}_{i}$ can be calculated by 


$$
\bar{M}\left(\sum_{i=1}^{n} x_{i} \widetilde{\varphi}_{i}\right)=\sum_{i=1}^{n} x_{i} \bar{M}\left(\widetilde{\varphi}_{i}\right)=\sum_{i=1}^{n} x_{i} \mu_{i} .
$$

From equations (5), (7) and (21), it can be deducted that

$$
\begin{aligned}
M_{U}\left(\widetilde{\varphi_{i}}\right) & =2 \int_{0}^{1} \gamma\left(\mu_{i}+\frac{\sqrt{2}}{2} \sigma_{i} \ln \sqrt{2} \sigma_{i} \gamma\right) d \gamma \\
& =\mu_{i}+\sqrt{2} \sigma_{i} \int_{0}^{1} \gamma \ln \sqrt{2} \sigma_{i} \gamma d \gamma \\
& =\mu_{i}+\frac{\sqrt{2}}{2} \sigma_{i}\left(\ln \sqrt{2} \sigma_{i}-\frac{1}{2}\right) .
\end{aligned}
$$

and

$$
\begin{aligned}
M_{L}\left(\widetilde{\varphi_{i}}\right) & =2 \int_{0}^{1} \gamma\left(\mu_{i}-\frac{\sqrt{2}}{2} \sigma_{i} \ln \sqrt{2} \sigma_{i} \gamma\right) d \gamma \\
& =\mu_{i}-\frac{\sqrt{2}}{2} \sigma_{i}\left(\ln \sqrt{2} \sigma_{i}-\frac{1}{2}\right) .
\end{aligned}
$$

In the same way, the following results can be obtained:

$$
\begin{aligned}
\sigma_{U}^{2} & =2 \int_{0}^{1} \gamma\left[M_{U}\left(\widetilde{\varphi_{i}}\right)-a_{2}(\gamma)\right]^{2} d \gamma \\
& =\frac{1}{8} \sigma_{i}^{2}
\end{aligned}
$$

and

$$
\begin{aligned}
\sigma_{L}^{2} & =2 \int_{0}^{1} \gamma\left[M_{U}\left(\widetilde{\varphi_{i}}\right)-a_{2}(\gamma)\right]^{2} d \gamma \\
& =\frac{1}{8} \sigma_{i}^{2}
\end{aligned}
$$

Thus, the possibilistic variance can be written as

$$
\overline{\sigma_{\widetilde{\varphi_{i}}}^{2}}=\frac{\sigma_{U}^{2}+\sigma_{L}^{2}}{2}=\frac{1}{8} \sigma_{i}^{2}
$$

Furthermore, according to equations (12) and (13), the upper and lower possibilistic covariances are given by

$$
\begin{array}{r}
\operatorname{Cov}_{U}\left(\widetilde{\varphi_{i}}, \widetilde{\varphi}_{j}\right)=2 \int_{0}^{1} \gamma\left[M_{U}\left(\widetilde{\varphi}_{i}\right)-a_{2}(\gamma)\right] \\
{\left[M_{U}\left(\widetilde{\varphi_{i}}\right)-b_{2}(\gamma)\right] d \gamma=\frac{1}{8} \sigma_{i} \sigma_{j},} \\
\operatorname{Cov}_{L}\left(\widetilde{\varphi_{i}}, \widetilde{\varphi}_{j}\right)=2 \int_{0}^{1} \gamma\left[M_{L}\left(\widetilde{\varphi_{i}}\right)-a_{1}(\gamma)\right] \\
{\left[M_{L}\left(\widetilde{\varphi_{i}}\right)-b_{1}(\gamma)\right] d \gamma=\frac{1}{8} \sigma_{i} \sigma_{j},}
\end{array}
$$

The possibilistic covariance is

$$
\begin{aligned}
\overline{\operatorname{Cov}}\left(\widetilde{\varphi_{i}}, \widetilde{\varphi_{j}}\right) & =\frac{\operatorname{Cov} U\left(\widetilde{\varphi_{i}}, \widetilde{\varphi_{j}}\right)+\operatorname{Cov}_{L}\left(\widetilde{\varphi_{i}}, \widetilde{\varphi_{j}}\right)}{2} \\
& =\frac{1}{8} \sigma_{i} \sigma_{j} .
\end{aligned}
$$

According to Lemma 2.2 and $\left(x_{i} \geq 0\right)$, we can compute the possibilistic variance of $\sum_{i=1}^{n} x_{i} \widetilde{\varphi_{i}}$

$$
\begin{array}{r}
\overline{\sum_{i=1}^{2} x_{i} \widetilde{\varphi_{i}}}=\sum_{i=1}^{n} x_{i}^{2} \overline{\sigma_{\widetilde{\varphi_{i}}}^{2}}+2 \sum_{i=1}^{n} x_{i} x_{j} \overline{\operatorname{Cov}}\left(\widetilde{\varphi_{i}}, \widetilde{\varphi}_{j}\right) \\
=\sum_{i=1}^{n} \frac{1}{8} x_{i}^{2} \sigma_{i}^{2}+2 \sum_{i=1}^{n} \frac{1}{8} x_{i} x_{j} \sigma_{i} \sigma_{j} \\
=\frac{1}{8}\left(\sum_{i=1}^{n} x_{i} \sigma_{i}\right)^{2} .
\end{array}
$$

So the proof of the theorem is complete.

According to Theorem 5.1 the membership function of $\sum_{i=1}^{n} x_{i} \widetilde{\varphi}_{i}$ is defined by:

$$
A(t)=\frac{1}{2 b} \exp \left\{-\frac{\left|t-\left(\sum_{i=1}^{n} x_{i} \mu_{i}\right)\right|}{b}\right\},
$$

where

$$
b=\frac{\sqrt{2}}{2} \sigma \Rightarrow b=\frac{\sqrt{2}}{2}\left(\frac{1}{8}\left(\sum_{i=1}^{n} x_{i} \sigma_{i}\right)^{2}\right) .
$$

So

$$
\operatorname{pos}\left(\sum_{i=1}^{n} \varphi_{i} x_{i} \leq V a R\right)=
$$

$$
\sup _{t \leq V a R}\left\{\frac{1}{2 b} \exp \left\{-\frac{\left|t-\left(\sum_{i=1}^{n} x_{i} \mu_{i}\right)\right|}{b}\right\}\right\} \text {, }
$$

$$
\begin{aligned}
& =\frac{1}{2 b} \exp \left\{-\frac{\left|V a R-\left(\sum_{i=1}^{n} x_{i} \mu_{i}\right)\right|}{b}\right\} . \\
& =\left\{\begin{array}{r}
\frac{1}{2 b} \exp \left\{-\frac{\sum_{i=1}^{n} x_{i} \mu_{i}-V A R}{b}\right\} i f, V a R<\sum_{i=1}^{n} x_{i} \mu_{i} \\
\frac{1}{2 b} \exp \left\{-\frac{V a R-\sum_{i=1}^{n} x_{i} \mu_{i}}{b}\right\} i f, V a R \geq \sum_{i=1}^{n} x_{i} \mu_{i}
\end{array}\right.
\end{aligned}
$$

According to equations (20) and (29), we can obtain 


$$
\begin{aligned}
& \left(V a R-\sum_{i=1}^{n} x_{i} \mu_{i}\right) \leq b \ln [2 b(1-\beta)] \text { if } V a R<\sum_{i=1} x_{i} \mu_{i} \\
& \left(\sum_{i=1}^{n} x_{i} \mu_{i}-V a R\right) \leq b \ln [2 b(1-\beta)] \text { if } V a R \geq \sum_{i=1} x_{i} \mu_{i} .
\end{aligned}
$$

It is known from equations (16), (24) and (17), that when the return rate of assets are Laplace distribution fuzzy variables, the upper and lower possibilistic means of $\widetilde{r_{p}}$ are given by:

$$
\begin{aligned}
& M_{U}\left(\widetilde{r_{p}}\right)=\sum_{i=1}^{n}\left(\mu+\frac{\sqrt{2}}{2} \sigma\left(\ln \sqrt{2} \sigma-\frac{1}{2}\right)-r_{f}\right) x_{i}+r_{f} \\
& M_{L}\left(\widetilde{r_{p}}\right)=\sum_{i=1}^{n}\left(\mu-\frac{\sqrt{2}}{2} \sigma\left(\ln \sqrt{2} \sigma-\frac{1}{2}\right)-r_{f}\right) x_{i}+r_{f}
\end{aligned}
$$

Thus, the possibilistic mean of $\widetilde{r}_{p}$ is written as

$$
\bar{M}\left(\widetilde{r_{p}}\right)=\frac{M_{U}\left(\widetilde{r_{p}}\right)+M_{L}\left(\widetilde{r_{p}}\right)}{2}=\sum_{i=1}^{n}\left(\mu_{i}-r_{f}\right) x_{i}+r_{f} .
$$

Moreover, for a Laplace fuzzy variable distribution, the portfolio model of VaR restrictions and risk free investment can be formulated as:

$$
\left\{\begin{array}{c}
\min \overline{\sigma^{2}}=\frac{1}{8}\left(\sum_{i=1}^{n} x_{i}^{2} \sigma_{i}^{2}+2 \sum_{i>j=1}^{n} x_{i} x_{j} \sigma_{i} \sigma_{j}\right) \\
\text { s.t. } \quad \sum_{i=1}^{n} x_{i}\left(\mu_{i}-r_{f}\right)+r_{f} \geq \widetilde{r} \\
\left(V a R-\sum_{i=1}^{n} x_{i} \mu_{i}\right) \leq b \ln [2 b(1-\beta)] p / V a R<\sum_{i=1}^{n} x_{i} \mu_{i} \\
\left(\sum_{i=1}^{n} x_{i} \mu_{i}-V a R\right) \leq b \ln [2 b(1-\beta)] p / V a R \geq \sum_{i=1}^{n} x_{i} \mu_{i} \\
\text { where } \quad b=\frac{\sqrt{2}}{16}\left(\sum_{i=1}^{n} x_{i} \sigma_{i}\right)^{2} \\
\sum_{i=1}^{n} x_{i} \leq 1 \\
0 \leq l_{i} \leq x_{i} \leq u_{i}, i=1,2, \ldots, n
\end{array}\right.
$$

\section{Numerical Example}

In order to compare the results using the three models of fuzzy distributions, namely, Normal and Laplace, whenever they are in the context of possibilistic mean-variance model described by Li et al, we selected three investment funds from Bradesco, using data from January to December of 2014. In a next step, we generated a database with 5290 possibilities $x_{i}, i=1,2, . ., n$, representing the proportion invested in assets $i$, namely, $0 \leq l_{i} \leq x_{i} \leq u_{i}, i=$ $1,2, \ldots, n$. For our work, we will consider $n=3$ assets and return rate for each asset $\widetilde{\varphi}_{i} \sim F N\left(\mu_{i}, \sigma_{i}\right)$ and $\widetilde{\varphi}_{i} \sim F L\left(\mu_{i}, \sigma_{i}\right)$, is calculated from the frequency distribution of months returns of the three assets, see Table 1.

Then we will analyze the results for each distribution function where $V a R=0.02$ and $\beta=0.9$ and the return of risk-free asset considered is $0.72 \%$.
Table 1: The possibilistic distributions of returns of three assets

\begin{tabular}{|c|c|c|c|}
\hline & \multicolumn{3}{|c|}{ Assets } \\
\hline & 1 & 2 & 3 \\
\hline$\mu$ & 0.7452 & 0.7035 & 0.8087 \\
$\sigma$ & 0.0319 & 0.0294 & 0.0278 \\
\hline
\end{tabular}

\subsection{Result for fuzzy Normal distribution}

In Figure 01, it was considered the return rate between $0.1 \%$ and $10 \%$ to the maximum risk rate of $10 \%$. We noted that the $3^{\text {rd }}$ possibility is one of the best choices for the investor, and investments would be distributed as follows: $1 \%$ on assets $01,1 \%$ on assets $02,10 \%$ in assets 03 and $88 \%$ risk-free.

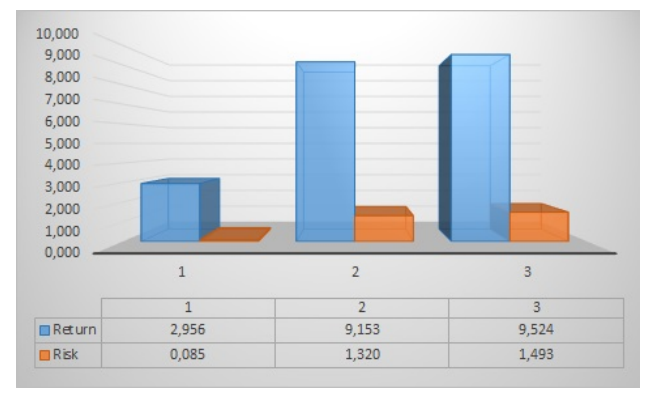

Figure 1: Result for Normal distribution with $V a R=0.02$ and $\beta=0.9$.

In Figure 02 we considered the return rate between $10 \%$ and $20 \%$ to the maximum risk rate of $10 \%$. We noted that the $7^{\text {th }}$ possibility is one of the best choices for the investor, and investments would be distributed as follows: $1 \%$ on assets $01,1 \%$ on assets $02,18.8 \%$ in assets 03 and $79.2 \%$ risk-free.

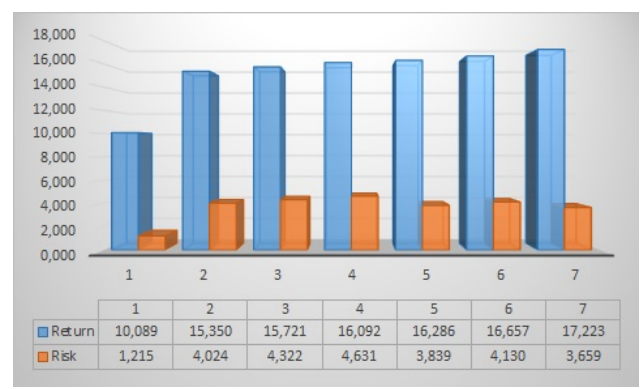

Figure 2: Result for Normal distribution with $V a R=0.02$ and $\beta=0.9$.

In Figure 03 we considered the return rate between $20 \%$ and $30 \%$ to the maximum risk rate of $10 \%$. We noted that the $10^{\text {th }}$ possibility is one of the best choices for the investor, and investments would be distributed as follows: $1 \%$ on assets 01 , $1 \%$ on assets $02,27.7 \%$ in assets 03 and $70.3 \%$ riskfree. 


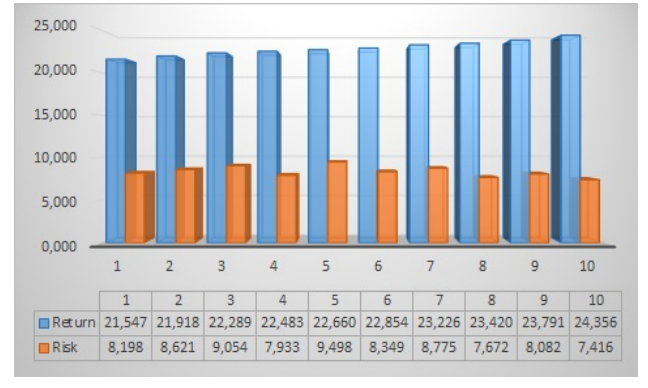

Figure 3: Result for Normal distribution with $V a R=0.02$ and $\beta=0.9$.

In Figure 04 we considered the return rate between $30 \%$ and $40 \%$ to the maximum risk rate of $20 \%$. We noted that the $8^{t h}$ possibility is one of the best choices for the investor, and investments would be distributed as follows: $1 \%$ on assets $01,1 \%$ on assets $02,45.5 \%$ in assets 03 and $52.5 \%$ risk-free.

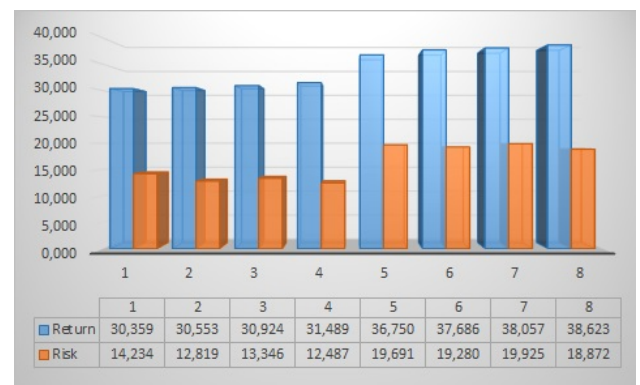

Figure 4: Result for Normal distribution with $V a R=0.02$ and $\beta=0.9$.

\subsection{Result for fuzzy Laplace distribution}

In Figure 05 we considered the return rate between $0.1 \%$ and $10 \%$ to the maximum risk rate of $10 \%$. We noted that the $3^{r d}$ possibility is one of the best choices for the investor, and investments would be distributed as follows: $1 \%$ on assets $01,1 \%$ on assets $02,9.9 \%$ in assets 03 and $88.1 \%$ risk-free.

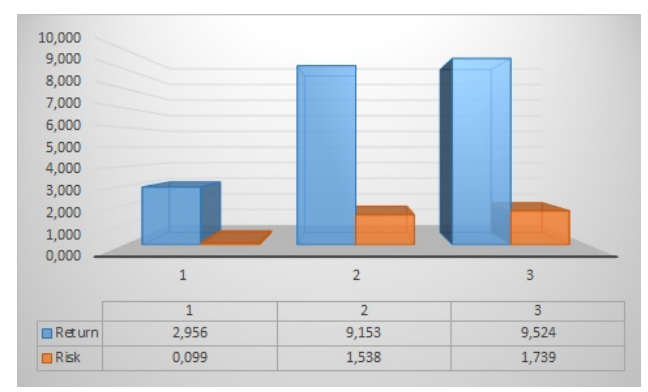

Figure 5: Result for Laplace distribution with $V a R=0.02$ and $\beta=0.9$.

In Figure 06 we considered the return rate between $10 \%$ and $20 \%$ to the maximum risk rate of $10 \%$. We noted that the $7^{\text {th }}$ possibility is one of the best choices for the investor, and investments would be distributed as follows: $1 \%$ on assets $01,1 \%$ on assets $02,18.8 \%$ in assets 03 and $79.2 \%$ risk-free.

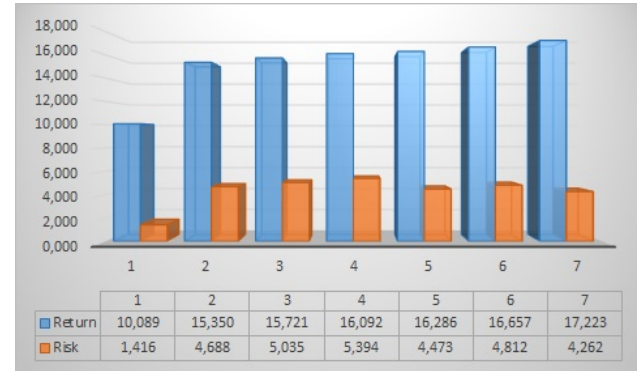

Figure 6: Result for Laplace distribution with $V a R=0.02$ and $\beta=0.9$.

In Figure 07 we considered the return rate between $20 \%$ and $30 \%$ to the maximum risk rate of $10 \%$. We noted that the $6^{\text {th }}$ possibility is one of the best choices for the investor, and investments would be distributed as follows: $1 \%$ on assets $01,1 \%$ on assets $02,27.7 \%$ in assets 03 and $70.3 \%$ risk-free.

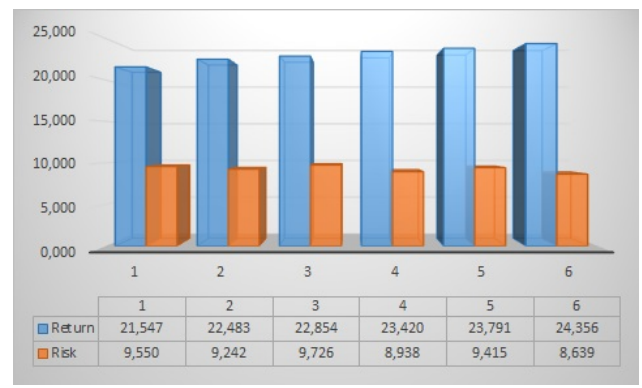

Figure 7: Result for Laplace distribution with $V a R=0.02$ and $\beta=0.9$.

In Figure 08 we considered the return rate between $30 \%$ and $40 \%$ to the maximum risk rate of $20 \%$. We noted that the $4^{t h}$ possibility is one of the best choices for the investor, and investments would be distributed as follows: $1 \%$ on assets $01,1 \%$ on assets $02,36.6 \%$ in assets 03 and $61.4 \%$ risk-free.

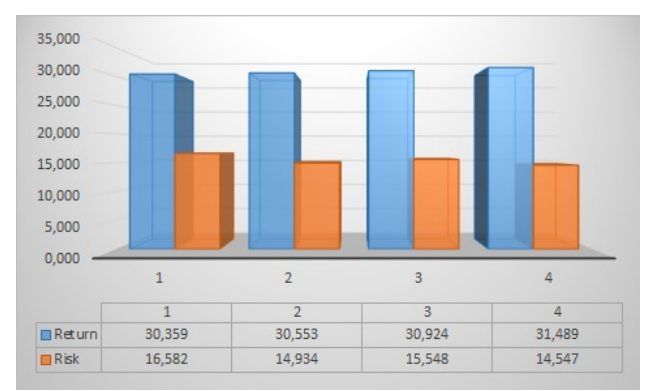

Figure 8: Result for Laplace distribution with $V a R=0.02$ and $\beta=0.9$.

In Figure 09 we presented the difference between the risk rates calculated by Normal distribution and Laplace distribution. Negative values indicate that the risk ratios calculated, when we use Laplace distribution is lower, but it is a small difference. 


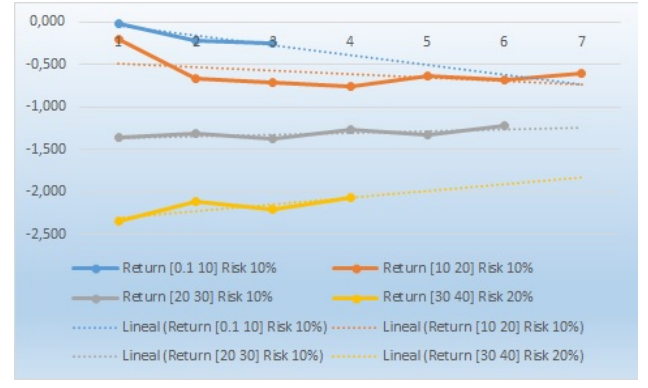

Figure 9: Result for Laplace distribution with $V a R=0.02$ and $\beta=0.9$.

\section{Conclusion}

This work compared models of fuzzy distribution, namely, Normal and Laplace, whenever they are inside the context of possibilistic mean-variance model described by $\mathrm{Li}$ et al. in [6], where fuzzy Normal distribution is used. We proposed to make a comparison using their model, but instead we applied fuzzy Laplace distribution. We also demonstrated the theorems which were necessary for the inclusion of these distributions to the model proposed by $\mathrm{Li}$ et al. So, we evaluated the behavior of this model when these distribution functions are changed and we also varied the VaR (Value at Risk). It is well known the importance of having other distributions as parameters for financial analysts, due the volatility of the stock market as well as the behavior of financial market.

We also observed the importance of working with several fuzzy probability distributions, as demonstrated by the significant variation in the return and risk rates. Besides, we demonstrated that for the model proposed by Li et al. the fuzzy Normal distribution and fuzzy Laplace distribution are the most appropriate ones.

Therefore, risk-averse investors were given the possibility of a better evaluation, i.e, deciding the best way to distribute their funds in assets investment.

\section{References}

[1] Alexander, G. J., Baptista, A. M., 2002. Economic implications of using a mean-VaR model for portfolio selection: a comparison with mean-variance analisys. Journal of Economic Dynamics and Control 26, 1159-1193.

[2] Carlsson, C., Fuller, R., 2001. On possibilistic mean value and variance of fuzzy numbers. Fuzzy Sets and Systems 122, 315-326.

[3] Duffie, D., Pan, J., 1997. An overview of value at risk. Journal of Derivatives 4, 7-49.

[4] Goestschel and Voxman, 1986. An overview of value at risk. Journal of Derivatives 4, 7-49.

[5] Leon, T., Liem, V., Vercher, E., 2002. Viability of infeasible portfolio selection problems: a fuzzy approach. European Journal of Operational Research 139, 178-189.

[6] Li, T., Zhang, W., Xu, W., 2013. Fuzzy possibilistic portfolio selection model with VaR constraint and risk-free investiment. Economic Modeling 31, 12-17.

[7] Markowitz,H., 1952. Portfolio selection. Journal of Finance 7, 77-91.

[8] Markowitz,H., 1959. Portfolio selection. Efficient diversification of Investments. Willey, New York.

[9] Ramaswamy, S.,1998. Portfolio selection using fuzzy decision theory. Working Paper of Bank for International Settlements, NÂ 59.

[10] Rocha, M. P. C. , Rocha, L. C. and Bedegral, B. C., 2014. Portfolio selection using Fuzzy distribution models with VaR constraint and risk free investment (2014).

[11] Tanaka, H., Guo, P., Turksen, I. B., 2000. Portfolio selection based on fuzzy probabilities and possibility distributions. Fuzzy Sets and Systems 111, 387-397.

[12] Wang, S. Y., Zhu, S. S., 2002. On fuzzy portfolio selection problems. Fuzzy Optimization and Decision Making 1, 361-377.

[13] Watada, J., 1997. Fuzzy portfolio selection and its applications to decision making. Tatra Mountains Mathematical Publication 13, 219248.

[14] Xu, W. D., Wu, C. F., Xu, W. J., Li, H.Y., 2010a. Dynamic asset allocation with jump risk. Journal of Risk 12, 29-44.

[15] Xu, W. J., Xu, W. D.,Li, H. Y., Zhang, W. G., 2010b. Uncertainty portifolio model in cross currency markets.International Journal of Uncertainty. Fuzziness and Knowledge-Based Systems 18, 759-777.

[16] Zdenec, Zmeskal, Z., 2005. Value at risk methodology of international index portfolio under soft conditions. International Review of Financial Analysis 14, 263-275.

[17] Zhang, W. G., Nie, Z. K., 2003. On possibilistic variance of fuzzy numbers. Lecture Notes in Artificial Intelligence 2639, 398-402.

[18] Zhang, W. G., Xiao, W. L., Xu, W. J., 2010. A possibilistic portifolio adjusting model with new added assets. Economic Modelling 27, 208213. 